RESENDE, G.M.; COSTA, N.D. Características produtivas da melancia em diferentes espaçamentos de plantio. Horticultura Brasileira, Brasília, v. 21, n. 4, p. 695-698, outubro-dezembro 2003.

\title{
Características produtivas da melancia em diferentes espaçamentos de plantio
}

\author{
Geraldo M. de Resende; Nivaldo D. Costa \\ Embrapa-Semi-Árido, C. Postal 23, 56300-000 Petrolina-PE; E-mail: gmilanez@ufla.br
}

\section{RESUMO}

Diferentes espaçamentos de plantio foram avaliados na produção de melancia em experimento da Embrapa Semi-Árido em Petrolina, de outubro a dezembro de 1998. Utilizou-se o delineamento experimental de blocos ao acaso em esquema fatorial $2 \times 3$, consistindo de dois espaçamentos entre linhas $(2,50$ e 3,00 m) e três espaçamentos entre plantas $(0,40 ; 0,60$ e $0,80 \mathrm{~m})$ e 3 repetições, sendo utilizada a cultivar Crimson Sweet. O espaçamento entre linhas de 3,00 $\mathrm{m}$ apresentou maior produção $(42,46 \mathrm{t} / \mathrm{ha})$, sendo que entre plantas os espaçamentos de 0,60 e $0,80 \mathrm{~m}$ alcançaram as maiores produções com 42,50 e 45,29 t/ha, respectivamente, não mostrando diferenças entre si. Não foram verificadas diferenças significativas para produção de frutos refugo nos espaçamentos entre linhas. No entanto, o menor espaçamento entre plantas proporcionou maior produção com $20,21 \mathrm{t} /$ ha, seguido pelos espaçamentos de 0,60 $\mathrm{m}(12,86 \mathrm{t} / \mathrm{ha})$ e $0,80 \mathrm{~m}(8,62 \mathrm{t} / \mathrm{ha})$. O incremento dos espaçamentos, tanto entre linhas como entre plantas, resultou em frutos de maior tamanho, tendo o espaçamento 3,00 x 0,80 m apresentado a maior massa fresca do fruto $(8,83 \mathrm{~kg} /$ fruto $)$. $\mathrm{O}$ maior número de frutos por planta (1,35 frutos) foi obtido com o espaçamento de $3,00 \times 0,80 \mathrm{~m}$.

Palavras-chave: Citrullus lanatus L., densidade de plantio, produção, massa fresca do fruto.

\section{ABSTRACT \\ Yield characteristics of watermelon in different planting spaces}

The yield of watermelon as a result of different planting space was evaluated, from October to December 1998 in Petrolina, Pernambuco State, Brazil. The experimental design was a randomized blocks in a $5 \times 3$ factorial scheme, with three replications. The cultivar Crimson Sweet was planted in 2.5 and $3.0 \mathrm{~m}$ row spacing and in 0.4 , 0.6 and $0.8 \mathrm{~m}$ inside the row. The $3.0 \mathrm{~m}$ row spacing resulted in the highest marketable yield with $42.46 \mathrm{tha}^{-1}$. Higher yields ( 42.50 and $45.29 \mathrm{t} \mathrm{ha}^{-1}$ ) were obtained with the 0.2 and $0.4 \mathrm{~m}$ plant spacing, with no difference between them. No significant differences were observed for unmarketable fruit yield in the row spacings. However, the smallest plant spacing $(0.4 \mathrm{~m})$ showed the highest yield with $20,21 \mathrm{tha}^{-1}$, followed by the $0.6 \mathrm{~m}\left(12.86 \mathrm{tha}^{-1}\right)$ and $0,80 \mathrm{~m}(8.62 \mathrm{t}$ $\left.\mathrm{ha}^{-1}\right)$. The increase of the row and plant spacing produced larger sized fruits, and the spacing $3.0 \times 0.8 \mathrm{~m}$ showed the highest weight of fresh fruit mass $(8.83 \mathrm{~kg})$. The largest number of fruits per plant (1.35 fruits) was observed in the $3.00 \times 0.8 \mathrm{~m}$ spacing.

Keywords: Citrullus lanatus L., plant density, yield, fresh fruit mass.

\section{(Recebido para publicação em 12 de fevereiro de 2003 e aceito em 18 de setembro de 2003)}

A melancia é originária da África Tropical, onde a forma selvagem é encontrada em muitos locais de clima tropical e subtropical, sendo o fruto redondo e pequeno com um diâmetro médio de $12 \mathrm{~cm}$ (Tessarioli Neto \& Groppo, 1992). Os frutos grandes e doces foram obtidos no antigo Egito, difundindo-se posteriormente para a Europa e Ásia. Embora também na Índia seja encontrada grande variabilidade desta espécie (Whitaker \& Davis, 1962).

Segundo a FAO (2003) a produção mundial em 2002 atingiu 89,9 milhões de toneladas e produtividade média de $25,1 \mathrm{t} / \mathrm{ha}$, tendo o Brasil produzido $620.000 \mathrm{t}$ com produtividade de 7,6 t/ha. Em 2002, a região Nordeste respondeu por $28,0 \%$ da produção do país, sendo os estados de Pernambuco e Bahia responsáveis por $65,9 \%$ desta produção (IBGE, 2003). A quantidade de melancia produzida no Brasil ocupa o quarto lugar den- tre as olerícolas, que têm produção anual em torno de 12,5 milhões de toneladas (Camargo Filho \& Mazzei, 2002).

No Nordeste o cultivo da melancia ocorre sob condições de chuva e sob irrigação. O primeiro é muito antigo, sendo em consórcio com outras culturas alimentares no período de dezembro a março. O cultivo em áreas irrigadas no Vale do São Francisco pode ocorrer durante todo o ano, sendo o período de agosto a outubro, o de maior concentração de plantio (Alvarenga \& Resende, 2002).

Em cucurbitáceas de forma geral, altas densidades produzem grande número de frutos por área, mas com tamanho, peso e número de frutos por planta reduzidos. Esse fato, segundo Robinson \& Walters (1997), tem sido atribuído principalmente às pressões de competição inter e intraplantas. Nas baixas densidades, tem sido verificado o inverso, ou seja, produção total menor com maior número de frutos por planta, de tamanho e peso mais elevado.

No tocante à densidade de plantio, as pressões exercidas pela população de plantas afetam de modo marcante o seu desenvolvimento. Quando aumenta a densidade de plantas por unidade de área, estas competem mais por fatores essenciais de crescimento, como nutrientes, luz e água (Janick, 1968).

As cultivares mais comercializadas no Brasil são Charleston Gray, Crimson Sweet, Fairfax, Omaru Yamato e Yamato Sato (Minami \& Iamauti, 1993), sendo que Carvalho (1997) ainda acrescenta as cultivares Pérola, Jubilee e Sunshade. Porém, Castellane \& Cortez (1995), citam que a 'Crimson Sweet' é a mais cultivada em todo o Brasil.

No Brasil, os espaçamentos mais utilizados nos plantios de melancia irrigados por aspersão são de 2,00 x 2,00 $\mathrm{m}$ para as cultivares de origem ameri- 
cana, e de 2,00 x 1,50 m, para as cultivares de origem japonesa, deixando-se duas plantas por cova (Casali et al., 1982). Já Sousa et al. (1995) relata 2,00 x 2,00 $\mathrm{m}$ para as cultivares de frutos cilíndricos e 2,00 x 1,50 m para as com frutos globulares, com duas plantas/ cova. Nos plantios irrigados por sulco ou por gotejamento, recomenda-se um espaçamento que pode variar de 2,50 a 3,00 x 0,70 a $1,00 \mathrm{~m}$, deixando-se apenas uma planta por cova. Plantios no final da estação chuvosa requerem espaçamentos mais arejados, considerando que as plantas apresentam maior desenvolvimento vegetativo e encurtamento do ciclo da cultura. Durante a estação seca, à medida que a temperatura torna-se mais amena, os espaçamentos podem ser mais próximos, considerando que o ciclo da cultura aumenta de 15 a 30 dias nesta época. Espaçamentos de 2,00 x 1,00 m em plantios nos meses de maio a julho são comuns na região de Uruana (GO), manejando-se uma planta por cova com um a dois frutos por planta (Miranda et al., 1997).

Para o estado de São Paulo o IAC (1998), recomenda que o plantio seja realizado em covas previamente preparadas e adubadas, com as dimensões médias de $0,40 \times 0,40 \times 0,30 \mathrm{~m}$, sendo os espaçamentos entre covas de 2,50 a $3,00 \times 2,00$ a $3,00 \mathrm{~m}$. Já Villa et al. (2001), relatam que o espaçamento pode variar de 2,50 a 3,00 x 1,50 a 2,00 m, dependendo da cultivar e das condições de cultivo, com um padrão de 3,0 × 2,0 m. Para as condições do Vale do São Francisco, Dias et al. (1998) e Soares et al. (1998), recomendam para plantio o espaçamento de $3,00 \mathrm{~m}$ x 0,80 a 0,70 $\mathrm{m}$, deixando uma planta por cova (4.166 a 4.762 plantas/hectare).

A planta de melancia possui considerável grau de elasticidade (Brinen et al., 1979) na qual, à medida que aumenta o espaço disponível para as plantas, aumenta o desenvolvimento e a produção de cada uma individualmente. Para as condições da Índia, Sing \& Naik (1989), relatam que os espaçamentos de 2,00 $\mathrm{x}$ $1,20 \mathrm{~m}$ e $2,00 \times 1,00 \mathrm{~m}$ foram os que proporcionaram as maiores produções e que a massa fresca do fruto aumentou com o incremento do espaçamento.

O melhoramento genético de melancia nos últimos anos tem enfatizado a qualidade do fruto em termos de teor de sólidos solúveis (brix), redução no número de sementes e tamanho. No Brasil, a preferência é por frutos graúdos, com peso superior a $6 \mathrm{~kg}$, que conseguem melhor cotação no mercado. Segundo Crall et al. (1994), nos Estados Unidos, o mercado consumidor tem dado preferência a híbridos diplóides e triplóides, de menor tamanho $(5-7 \mathrm{~kg})$, e frutos tipo "ice-box ", redondos e pequenos de 1 a $5 \mathrm{~kg}$, de polpa vermelha ou amarela, também conhecidas como melancias de geladeira (PUC, 2001).

Frutos de melancia são classificados de acordo com o peso, em grandes ( $>9$ $\mathrm{kg})$, médios (6-9 kg) e pequenos $(<6 \mathrm{~kg})$, onde aqueles com peso acima de $7 \mathrm{~kg}$ obtêm os melhores preços (Alvarenga \& Resende, 2002).

O objetivo deste trabalho foi estabelecer a densidade de plantio mais adequada para o cultivo da melancia cultivar Crimson Sweet no Vale do São Francisco, visando maior produção e qualidade dos frutos para os mercados interno e externo.

\section{MATERIAL E MÉTODOS}

O ensaio foi instalado em latossolo na Embrapa Semi-Árido, Petrolina, cujas coordenadas geográficas são $9^{\circ} 9^{\prime}$ de latitude Sul e $40^{\circ} 29^{\prime}$ de longitude Oeste e altitude de $365,5 \mathrm{~m}$, no período de outubro a dezembro de 1998.

Utilizou-se o delineamento experimental de blocos ao acaso em esquema fatorial $2 \times 3$, consistindo em dois espaçamentos entre linhas $(2,50$ e 3,00 m) e três espaçamentos entre plantas $(0,40 ; 0,60$ e $0,80 \mathrm{~m})$ e 3 repetições. As parcelas foram constituídas de 4 linhas de 7,20 m, sendo as duas linhas centrais consideradas como área útil.

A adubação de plantio de foi feita com $30 \mathrm{~kg} /$ ha de $\mathrm{N}, 120 \mathrm{~kg} / \mathrm{ha}$ de $\mathrm{P}_{2} \mathrm{O}_{5} \mathrm{e}$ $60 \mathrm{~kg} / \mathrm{ha}$ de $\mathrm{K}_{2} \mathrm{O}$ e a cobertura, em número de duas, na dose de $30 \mathrm{~kg} / \mathrm{ha}$ de $\mathrm{N}$ e $\mathrm{K}_{2} \mathrm{O}$ aplicados aos 20 e 40 dias após a semeadura (Cavalcanti, 1998).

A irrigação foi feita por aspersão convencional, sendo a cultura mantida no limpo por meio de capinas manuais e os demais tratos fitossanitários os comumente empregados na cultura.
Utilizou-se a cultivar Crimson Sweet, plantada em 02/10/98, quando foram colocadas três a quatro sementes por cova. No desbaste, quinze dias após a semeadura deixou-se uma planta por cova.

A colheita foi realizada aos 70 dias após a semeadura e sete dias após a primeira. Avaliou-se a produção de frutos comerciais em $\mathrm{t} / \mathrm{ha}$ (frutos com mais de $6 \mathrm{~kg}$ ) e refugos (frutos abaixo de $6 \mathrm{~kg}$, pequenos e deformados), massa fresca do fruto ( $\mathrm{kg} /$ fruto), número de frutos por planta e porcentagem de frutos comerciais e não comerciais (refugos). Posteriormente, foi feita a análise de variância das características avaliadas, aplicandose o teste de Tukey ao nível de 5\% de probabilidade para comparação das médias. Os dados de porcentagem foram previamente transformados em $\operatorname{arco-seno} \sqrt{P / 100}$, para efeitos de análise estatística, sendo apresentados os dados originais.

\section{RESULTADOS E DISCUSSÃO}

Houve efeitos significativos independentes para espaçamento entre linhas e entre plantas e ainda para a interação entre estes fatores.

A produção comercial teve efeitos significativos independentes entre os fatores estudados (Tabela 1). O espaçamento de 3,00 m entre linhas apresentou maior produção $(42,46 \mathrm{t} / \mathrm{ha})$, diferindo significativamente de $2,50 \mathrm{~m}$, onde os espaçamentos de 0,60 e $0,80 \mathrm{~m}$ entre plantas alcançaram as maiores produções (42,50 e 45,29 t/ha, respectivamente), não mostrando diferenças entre si. No entanto, com a redução do espaçamento para $0,40 \mathrm{~m}$ entre plantas verificou-se menor produção com 34,79 t/ha. Estes resultados são superiores à média mundial e nacional relatada pela FAO (2003), assim como estão próximos às recomendações de Dias et al. (1998) e Soares et al. (1998), que sugerem para a região o espaçamento de 3,00 m x 0,80 a $0,70 \mathrm{~m}$, deixando uma planta por cova (4.166 a 4.762 plantas/hectare).

Efeitos significativos independentes dos fatores estudados também foram observados para produção de frutos refugo e porcentagem de frutos comerciais. As demais características avalia- 
Tabela 1. Produção comercial e refugo e porcentagem de frutos comerciais em função de diferentes espaçamentos. Petrolina, Embrapa Semi-Árido, 1998.

\begin{tabular}{lccc}
\hline \multirow{2}{*}{ Espaçamento $(\mathbf{m})$} & \multicolumn{2}{c}{ Produção (t/ha) } & $\begin{array}{c}\text { Porcentagem de frutos } \\
\text { comerciais (\%) }\end{array}$ \\
\cline { 2 - 3 } Entre linhas & Comercial & Refugo & \\
\hline 2,50 & & & $75,67 \mathrm{a}$ \\
3,00 & $39,25 \mathrm{~b}$ & $14,39 \mathrm{a}$ & $72,76 \mathrm{a}$ \\
\hline Entre plantas & $42,46 \mathrm{a}$ & $13,40 \mathrm{a}$ & $81,19 \mathrm{a}$ \\
\hline 0,80 & & & $74,35 \mathrm{~b}$ \\
0,60 & $45,29 \mathrm{a}$ & $8,62 \mathrm{c}$ & $66,47 \mathrm{c}$ \\
0,40 & $42,50 \mathrm{a}$ & $12,86 \mathrm{~b}$ & 3,86 \\
\hline C.V. $(\%)$ & $34,79 \mathrm{~b}$ & $20,21 \mathrm{a}$ & 11,55 \\
\hline
\end{tabular}

*Médias seguidas pela mesma letra minúscula na coluna, não diferem entre si pelo teste de Tukey ao nível de 5\% de probabilidade.

Tabela 2. Massa fresca dos frutos, número de frutos por planta e porcentagem de frutos não comerciais em função de diferentes espaçamentos. Petrolina, Embrapa Semi-Árido, 1998.

\begin{tabular}{|c|c|c|c|}
\hline \multicolumn{4}{|c|}{ Massa fresca dos frutos $(\mathrm{kg})$} \\
\hline \multirow{2}{*}{ Espaçamentos entre linhas $(m)-$} & \multicolumn{3}{|c|}{ Espaçamentos entre plantas (m) } \\
\hline & 0,80 & 0,60 & 0,40 \\
\hline 2,50 & $7,84 \mathrm{~b} \mathrm{~A}$ & $7,34 \mathrm{~b} \mathrm{~B}$ & $6,91 \mathrm{~b} \mathrm{C}$ \\
\hline \multirow[t]{2}{*}{3,00} & 8,83 a $A$ & 8,08 a $B$ & 7,33 a $C$ \\
\hline & \multicolumn{3}{|c|}{ Número de frutos por planta } \\
\hline 2,50 & $1,12 \mathrm{~b} \mathrm{~A}$ & $0,93 \mathrm{~b} \mathrm{~B}$ & 0,66 a C \\
\hline \multirow[t]{2}{*}{3,00} & 1,35 a $A$ & 1,11 a $B$ & 0,63 a $C$ \\
\hline & \multicolumn{3}{|c|}{ Porcentagem de frutos não comerciais (\%) } \\
\hline 2,50 & 22,16 a $B$ & 25,40 a $B$ & 34,89 a $A$ \\
\hline 3,00 & 14,42 b B & 25,90 a $A$ & 32,13 a $A$ \\
\hline
\end{tabular}

Médias seguidas de mesma letra minúscula nas colunas e maiúsculas nas linhas não diferem entre si a 5\% de probabilidade pelo teste de Tukey.

das apresentaram interação entre os fatores estudados. Não foram verificadas diferenças significativas para produção de frutos refugo entre os espaçamentos entre linhas (Tabela 1). No entanto, o menor espaçamento entre plantas proporcionou maior produção com 20,21 t/ ha, seguido pelos espaçamentos de 0,60 $\mathrm{m}(12,86 \mathrm{t} / \mathrm{ha})$ e $0,80 \mathrm{~m}(8,62 \mathrm{t} / \mathrm{ha})$. Este fato foi decorrente do maior número de frutos pequenos (abaixo de $6 \mathrm{~kg}$ ) apresentado pelo espaçamento de $0,40 \mathrm{~m}$. Também, Brinen et al. (1979) observaram resultado semelhante com o aumento da densidade. A porcentagem de frutos não comerciais aumentou quando se reduziu o espaçamento, tanto entre linhas como entre plantas (Tabela 2). Observa-se que somente houve diferenças significativas entre linhas, quando se utilizou o espaçamento entre plantas de $0,80 \mathrm{~m}$. Para o espaçamento de 2,50 m entre linhas não verificou-se diferenças quando se usou o espaçamento de 0,60 e $0,80 \mathrm{~m}$ entre plantas. Assim como a produção de frutos refugo, a porcentagem de frutos comerciais (Tabela 1) não evidenciou efeitos sobre o espaçamento entre linhas. Ao contrário, a produção de frutos comerciais foi incrementada quando a densidade de plantio se reduziu, ou seja, a maior porcentagem de frutos comerciais foi alcançada no espaçamento de $0,80 \mathrm{~m}$ (81,08\%). Sanders et al. (1999), relata um decréscimo na produção de frutos comerciais por hectare com a diminuição do espaçamento entre linhas. Em cucurbitáceas de forma geral, altas densidades produzem grande número de frutos por área, mas com tamanho, peso e número de frutos por planta reduzidos. Este fato tem sido atribuído principalmente às pressões de competição inter e intraplantas (Robinson \& Walters, 1997).

Constatou-se efeito significativo da interação entre os fatores estudados para massa fresca do fruto e número de frutos por planta (Tabela 2). O incremento dos espaçamentos tanto entre linhas como entre plantas produziu frutos de maior tamanho, tendo o espaçamento $3,00 \times 0,80 \mathrm{~m}$ apresentado a maior massa fresca de fruto $(8,83 \mathrm{~kg} /$ fruto). Resultados similares foram observados por Nesmith (1993) que verificou maior massa fresca do fruto no maior espaçamento entre plantas para a mesma cultivar, assim como por Sing \& Naik (1989) e Sanders et al. (1999), que relataram maior massa fresca do fruto com o incremento do espaçamento. Outros relatos semelhantes foram encontrados da competição promovendo uma redução na massa fresca dos frutos com o 
aumento da densidade de plantio (Firbank \& Watkinson, 1990; Weiner, 1990).

Com relação ao circuito de comercialização, verifica-se que no mercado interno, os frutos preferidos são os maiores, com peso acima de $7 \mathrm{~kg}$, e são também os de maior cotação de mercado (Alvarenga \& Resende, 2002).

$\mathrm{O}$ número frutos por planta foi superior nos maiores espaçamentos entre linhas e entre plantas, exceto no espaçamento de $0,40 \mathrm{~m}$ que não mostrou diferenças significativas tanto nos espaçamentos de 3,00 ou de 2,50 m entre linhas (Tabela 2). O maior número de frutos por planta (1,35 frutos) foi apresentado pelo espaçamento $3,00 \mathrm{x}$ $0,80 \mathrm{~m}$. Resultados estes concordantes com Duthie et al. (1999) que observaram maior número de frutos comerciais por planta em menores densidades. $\mathrm{O}$ menor número de frutos por planta, com o aumento da densidade de plantio, devese possivelmente à menor disponibilidade de espaço entre plantas, que promove maior competição por elementos nutritivos, água e luz e conseqüentemente diminuição da atividade fotossintética, produção de ramificações, folhas, flores e processo de polinização através das abelhas (principais responsáveis pela fecundação dos frutos). Quando a densidade de plantas aumenta por unidade de área, atinge-se um ponto no qual as plantas competem por fatores essenciais de crescimento, como nutrientes, luz e água (Janick, 1968).

Estes resultados evidenciam ser a densidade de plantio uma ferramenta de grande importância para os agricultores, visto que, em função da demanda do mercado consumidor, pode-se manejar com maior ou menor densidade de plantas, visando maior retorno econômico e maximizando a produção. Em função dos resultados obtidos, recomenda-se o espaçamento de 3,00 × 0,60 a $0,80 \mathrm{~m}$ para as condições do Vale do São Francisco. No entanto, levando-se em conta que os mercados interno e externo ten- dem a optar por frutos de menor massa fresca, o espaçamento de $3,00 \times 0,40 \mathrm{~m}$ poderá ser indicado em função da maior produção de frutos pequenos e que na atual classificação foram definidos como não comerciais (refugos) por apresentarem massa fresca do fruto abaixo de $6,0 \mathrm{~kg} /$ fruto.

\section{LITERATURA CITADA}

ALVARENGA, M.A.R.; RESENDE, G.M. Cultura da melancia. Lavras: Editora UFLA, 2002. 132 p. (UFLA, Textos Acadêmicos, 19).

BRINEN, G.H.; LOCASCIO, S.J.; ELMSTROM, G.W. Plant and row spacing, mulch, and fertilizer rate effects on watermelon production. Journal American Society Horticultural Science, v. 104, n. 6, p. 724-726, 1979.

CAMARGO FILHO, W.P.; MAZZEI, A.R. O mercado de melancia no Mercosul. Informações Econômicas, São Paulo, v. 32, n. 2, 2002.

CARVALHO, R.N. Cultivo da melancia para a agricultura familiar. Brasília: EMBRAPA - SPI, 1999. $127 \mathrm{p}$.

CASALI, V.W.O.; SONNEnBERG, P.E.; PEDROSA, J.F. Melancia: cultivares e métodos culturais. Informe Agropecuário, Belo Horizonte, v. 8, n. 85, p. 29 - 32, 1982.

CASTELLANE, P.D.; CORTEZ, G.E. A cultura da melancia. Jaboticabal: FUNEP, 1995. 64 p.

CAVALCANTI, F.J.A., coord. Recomendações de adubação para o Estado de Pernambuco: $2^{\mathrm{a}}$ aproximação. Recife: IPA. 1998. 198 p.

CRALL， J.M.; ELMSTROM， G.W.; MCCUISTION Jr., F.T. SSdl: a high quality icebox watermelon breeding line resistant to Fusarium wilt and antracnose. HortScience, v. 29, n. 6, p. 707-711, 1994.

DIAS, R.C.S.; COSTA, N.D.; QUEIROZ, M.A.; RESENDE, G.M.; ALVES, R.; OLIVEIRA, C.A.V. Densidade de plantio em melancia. In: CONGRESSO BRASILEIRO DE OLERICULTURA, 38, Petrolina-PE, 1998. Horticultura Brasileira, Brasília, v. 16, n. 1, 1998. (Resumo, 091).

DUTHIE, J.A.; ROBERTS, B.W.; EDELSON, J.V.; SHREFLER, J.W. Plant density-dependent variation in density, frequency, and size of watermelon fruits. Crop Science, v. 39, n. 2, p. 406-412, 1999.

FAO (Roma, Italy). Agricultural production, primary crops. Disponível em $<\mathrm{http}: / /$ www.fao.org $>$. Acesso em 29/01/03.

FIRBANK, L.G.; WATKINSON, A.R. One effects of competition: from monocultures to mixtures. In: GRACE, J. B. (ed.) Perspectives on plant competition. San Diego: Academic Press, 1990. p. 165-192.
IAC. Instruções agrícolas para as principais culturas econômicas. $6^{\text {a }}$ ed. Campinas: IAC, 1998, 396 p. (IAC. Boletim, 200).

IBGE. Indicadores conjunturais - produção agrícola/agricultura. Disponível em <http:// www.Ibge.gov.br>. Acesso em 29/01/03.

JANICK, J. A Ciência de horticultura. 2.ed. Rio de Janeiro: Freitas Bastos, 1968. 485 p.

MINAMI, K.; IAMAUTI, M.J. Cultura da melancia. Piracicaba: ESALQ/USP, 1993. 101 p. MIRANDA, R.F; RODRIGUES, G.A; SILVA, R.H; SILVA, C.L.W; SATURNINO, H.M.; FARIA, S.H.F. Instruções técnicas sobre a cultura da melancia. Belo Horizonte: EPAMIG, 1997. 28 p. (EPAMIG. Boletim Técnico, 51).

NESMITH, D.S. Plant spacing influences watermelon yield and yield components. HortScience, v. 28, n. 9, p. 885-887, 1993.

PUC (Chile). Hortalizas. Disponível em $<$ http:www.puc.cl/sw-educ/hortalizas/ html/ sandia/diversidad-sandia. html>. Acesso em 08/ 04/01.

ROBINSON, R.W.; WALTERS, D.S.D. Cucurbits. New York: CAB International, 1997. 226 p.

SANDERS, D.C.; CURE, J.D.; SCHULTHEIS, J.R. Yield response of watermelon to planting density, planting pattern, and polyethylene mulch. HortScience, v. 34, n. 7, p. 1221-1223, 1999.

SING, R.V.; NAIK, L.G. Response of watermelon (Citrullus lanatus Thumbs. Monsef.) to plant density, nitrogen and phosphorus fertilization. Indian Journal Horticulture, v. 46, n. 1, p. 80-83, 1989.

SOARES, J.M.; BRITO, L.T.L.; RESENDE, G.M.; CHOUDHURY, M.M. Níveis de nitrogênio via água de irrigação e densidade de plantio na cultura da melancia. In: CONGRESSO BRASILEIRO DE OLERICULTURA, 38, Petrolina, 1998. Horticultura Brasileira, Brasília, v. 16, n. 1, 1998. (Resumo, 326).

SOUSA, V.A.B.; VIANA, F.M.P.; BARRIGOSSI, J.A.F. Informações técnicas para o cultivo da melancia no Piauí. Teresina: EMBRAPA CPAMN, 1995. 36 p. (EMBRAPA - CPAMN. Circular Técnica, 14).

TESSARIOLI NETO, J.; GROPPO, G.A.A. Cultura da melancia. Campinas: CATI, 1992. 11 p. (Boletim Técnico, 242).

VILLA, W.; GROPPO, G.A.; TESSARIOLI NETO, J; GELMINI, G.A. Cultura da melancia. Campinas: CATI, 2001. 52 p. (CATI. Boletim técnico, 243).

WEINER, J. Plant population ecology in agriculture. In: CARROL, C.R. et al. (eds.) Agroecology. New York: McGraw Hill, 1990. p. 235-262.

WHITAKER, T.W.; DAVIS, G.N. Cucurbits. New York: Interscience Publishers, 1962, 250 p. 